\title{
Structure and expression of the fliA operon of Salmonella typhimurium
}

\author{
Tadayoshi Ikebe, Sunao lyoda† and Kazuhiro Kutsukake
}

Department of Applied Biochemistry, Faculty of Applied Biological Science, Hiroshima University, Kagamiyama 1-4-4, HigashiHiroshima, Hiroshima 739-8528, Japan

\author{
Author for correspondence: Kazuhiro Kutsukake. Tel: +81 82424 7924. Fax: + 81824247925. \\ e-mail: ktkk@ipc.hiroshima-u.ac.jp
}

\begin{abstract}
The fliA gene encodes the flagellum-specific sigma factor $\sigma^{28}$ in Salmonella typhimurium. The transcription in vivo and in vitro of this gene was analysed and it was found that there are two promoters for the expression of this gene. One is a class 2 promoter which is recognized by $\sigma^{70}$-RNA polymerase in the presence of the FlhD and FlhC activator proteins. The other is a class 3 promoter which is recognized by $\sigma^{28}$-RNA polymerase. Therefore, the fliA operon is under dual positive control from FlhD/FlhC and from FliA itself. The nucleotide sequence downstream of the fliA gene was determined. The sequence contains two ORFs following the fliA gene. On the basis of their sequence homology, it is concluded that these two correspond to the fliz and fliY genes of Escherichia coli. Northern blot analysis revealed that the fliz gene is transcribed from the fliA promoters, whereas the fliY gene is transcribed from both the fliA promoters and its own FlhD/FlhC-independent promoter. A fliz-disruption mutant was constructed by inserting a kanamycin-resistance gene cassette into the fliz gene on the chromosome. The mutant showed poor motility, and introduction of a fliz ${ }^{+}$plasmid into this mutant restored the wildtype level of motility. These results suggest that the fliz gene may be required for expression of maximal motility.
\end{abstract}

Keywords: Salmonella, flagellum-specific sigma factor, primer extension, in vitro transcription, gene disruption

\section{INTRODUCTION}

The flagellum of Salmonella typhimurium is composed of three structural parts, a basal body, a hook and a filament, and is constructed in this order (Macnab, 1996). More than 50 genes are required for flagellar formation and function. These flagellar genes constitute at least 14 different operons, and most of them are clustered in four regions on the chromosome (Kutsukake et al., 1988; Macnab, 1996). Transcription of these operons forms a highly organized cascade called the flagellar regulon, and is coordinated with the flagellar assembly hierarchy (Kutsukake et al., 1990; Kutsukake $\&$ Iino, 1994). According to the relative positions in the transcriptional hierarchy, the flagellar operons are grouped into three classes, 1,2 and 3 . Class 1 contains only one operon, $f h D D$, consisting of two genes, $f l h D$ and

\footnotetext{
†Present address: Department of Bacteriology, National Institute of Infectious Diseases, Toyama 1-23-1, Shinjuku-ku, Tokyo 162-8640, Japan.

The DDBJ/EMBLGenBank accession number for the sequence data reported in this paper is AB010947.
}

flhC, whose products are both required for class 2 expression. Class 2 contains the operons responsible for formation of the flagellar basal structure, the hookbasal body complex. Class 2 also contains the fliA gene, which encodes an alternative sigma factor, $\sigma^{28}$, needed for class 3 expression (Ohnishi et al., 1990). Class 3 contains operons responsible for filament formation, flagellar rotation and chemotaxis. FliA-dependent expression of class 3 is under negative control from an anti-sigma factor, FlgM (Gillen \& Hughes, 1991; Ohnishi et al., 1992; Kutsukake \& Iino, 1994; Kutsukake et al., 1994b; Iyoda \& Kutsukake, 1995; Chadsey et al., 1998). The flgM gene is expressed from both class 2 and class 3 promoters (Gillen \& Hughes, 1993; Kutsukake, 1994; Kutsukake et al., 1994a). In addition to class 3 expression, the FliA-FlgM regulatory system is known to be involved in regulation of class 1 and class 2 expression (Kutsukake \& Iino, 1994; Kutsukake, 1997b). However, a molecular mechanism underlying this control has remained unknown.

Escherichia coli contains a similar number of flagellar genes, and their expression is organized in a similar 
Table 1. Bacterial strains and plasmids used

\begin{tabular}{|c|c|c|}
\hline Strain/plasmid & Relevant characteristics & Source/reference \\
\hline \multicolumn{3}{|l|}{ Strains } \\
\hline \multicolumn{3}{|l|}{ Escherichia coli } \\
\hline BL21(DE3) & T7 expression host & Studier \& Moffatt (1986) \\
\hline EKK22 & hsd flhD & Ohnishi et al. (1990) \\
\hline \multicolumn{3}{|c|}{ Salmonella typhimurium } \\
\hline KK1004 & Wild-type & Kutsukake et al. (1988) \\
\hline KK1397 & fliZ::kan & This study* \\
\hline KK2040 & $f l b D:: \operatorname{Tn} 10$ & Kutsukake et al. (1988) \\
\hline KK2091 & $f l i A:: \operatorname{Tn} 10$ & Kutsukake et al. (1988) \\
\hline \multicolumn{3}{|l|}{ Plasmids } \\
\hline pACYC184 & Cloning vector, $\mathrm{Cm}^{\mathrm{r}} \mathrm{Tc}^{\mathrm{r}}$ & Chang \& Cohen (1978) \\
\hline pBR322 & Cloning vector, $A p^{r} T c^{r}$ & Bolivar et al. (1977) \\
\hline $\mathrm{pET} 17 \mathrm{~b}$ & $\mathrm{~T} 7$ expression vector, $\mathrm{Ap}^{\mathrm{r}}$ & Novagen \\
\hline pSTV29 & Cloning vector, $\mathrm{Cm}^{\mathrm{r}}$ & Takara \\
\hline pTrc97A & tac expression vector, $\mathrm{Ap}^{\mathrm{r}}$ & Amann et al. $\langle 1988\rangle$ \\
\hline pTrc99C & tac expression vector, $\mathrm{Ap}^{\mathrm{r}} \operatorname{lacl}^{\mathrm{q}}$ & Amann et al. (1988) \\
\hline pUC4K & kan gene cassette, $\mathrm{Ap}^{\mathrm{r}}$ & Pharmacia \\
\hline pUC19 & Cloning vector, $\mathrm{Ap}^{\mathrm{r}}$ & Yanisch-Perron et al. (1985) \\
\hline pKK1059 & pBR322 fliAZY $Y^{+}$ & Ohnishi et al. $(1990) \dagger$ \\
\hline pKK1064 & pBR322 $f l i A^{+}$ & Ohnishi et al. $(1990) \dagger$ \\
\hline pSIIA1 & $\mathrm{pTrc} 99 \mathrm{C} \mathrm{fli}^{+}$ & Kutsukake et al. (1994b)† \\
\hline pSIIA100 & $\mathrm{p} \operatorname{Trc} 97 \mathrm{~A} \Delta(t a c) \mathrm{p}_{f l i A^{-}} \mathrm{t}_{r r n B}$ & S. Iyoda, unpublished $\dagger$ \\
\hline pSIHD1 & $\mathrm{pET} 17 \mathrm{~b} f l b D^{+}$ & S. Iyoda, unpublished \\
\hline pSIHC2 & pACYC184 $\mathrm{flbC}^{+}$ & S. Iyoda, unpublished \\
\hline pBRIA2 & $\mathrm{pBR} 322 \mathrm{p}_{\text {fliA }}$ & This study \\
\hline pUCIZ1 & pUC19 fliZ' & This study* \\
\hline pKKIZ2 & pUCIZ1 fliZ' (PvuII) 'fliZ & This study* \\
\hline pKKIZ3 & pUCIZ2 fliZ::kan & This study*† \\
\hline pTIIZ2 & pSTV29 fliZ ${ }^{+}$ & This studyt \\
\hline
\end{tabular}

* Construction procedures are described in detail in Methods.

†S. typhimurium chromosomal regions carried by these plasmids are shown in Fig. 1(b).

cascade fashion (Komeda et al., 1980; Komeda, 1982, 1986). The FlhD and FlhC proteins of E. coli were purified and shown to act as the activators for $\sigma^{\mathbf{7 0}}$ dependent expression of the class 2 operons (Liu \& Matsumura, 1994). Like FliA of S. typhimurium, FliA of $E$. coli was shown to be an alternative sigma factor specific for flagellar operons (Liu \& Matsumura, 1995). Mytelka \& Chamberlin (1996) reported that the fliA gene constitutes an operon together with two genes, $f l i Z$ and $f l i Y$, in E. coli. They postulated that these two genes may not be essential for motility but have a regulatory role. Expression of the $f l i A$ operon of $E$. coli was characterized in vivo and in vitro, and was shown to be under dual control from class 2 and class 3 promoters (Mytelka \& Chamberlin, 1996; Liu \& Matsumura, 1996). Therefore, the fliA operon of E. coli is transcribed both by $\sigma^{70}$-RNA polymerase in the presence of FlhD and FlhC and by $\sigma^{28}$-RNA polymerase.

Though the fliA gene product has been characterized extensively in S. typhimurium (Ohnishi et al., 1990; Kutsukake et al., 1994b), the entire structure and expression of the fliA operon have been less wellcharacterized in this organism. In the present work, we analysed the DNA sequence and transcriptional control of the fliA operon of S. typhimurium. As in E. coli, the $f l i A$ operon was found to contain two additional genes, $f l i Z$ and $f l i Y$, and to be transcribed from both the class 2 and class 3 promoters. However, we obtained evidence suggesting that the $f l i$ gene is also transcribed from its own non-flagellar promoter. A disruption mutant was constructed by inserting a kanamycin-resistance gene cassette into the fliZ gene on the chromosome. The mutant showed poor motility, and maximal motility was recovered by introducing a $\mathrm{fliZ}^{+}$plasmid into this mutant. This suggests that the fliZ gene, though not essential, is required for expression of maximal motility.

\section{METHODS}

Bacterial strains, plasmids and media. Bacterial strains and plasmids used in the present study are listed in Table 1. Ordinary culture media including L broth, L-agar plates, motility agar plates and minimal medium were prepared as 
described previously (Kutsukake, 1997a). Ampicillin, chloramphenicol and kanamycin were used at final concentrations of 50,25 and $25 \mu \mathrm{g} \mathrm{ml}^{-1}$, respectively. In order to induce genes fused to the tac promoter on the expression plasmids, IPTG was added to a final concentration of $1 \mathrm{mM}$.

Oligonucleotide primers, chemicals and enzymes. Customized primers were purchased from Life Technologies, Kurabo or Pharmacia. Unless otherwise specified, all the chemicals were purchased from Nacalai Tesque. E. coli RNA polymerase core enzyme and holoenzyme were purchased from Epicentre Technologies and Boehringer Mannheim, respectively. Taq DNA polymerase and Moloney murine leukaemia virus reverse transcriptase were purchased from Promega and Amersham, respectively. Other DNA-modifying enzymes were purchased from Toyobo or Nippon Gene.

Protein purification. The S. typhimurium FliA protein formed inclusion bodies in the IPTG-treated cells of EKK22 harbouring pSIIA1. From these inclusion bodies, FliA was purified according to the purification procedure for E. coli $\sigma^{70}$ developed by Igarashi \& Ishihama (1991). The S. typhimurium FlhD/FlhC complex was purified from the soluble fraction of the IPTG-treated cells of BL21(DE3) carrying both pSIHD1 and $\mathrm{pSIHC} 2$ according to the purification procedure for the $E$. coli FlhD/FlhC complex described by Liu \& Matsumura (1994).

Primer extension analysis. Total RNA was isolated from S. typhimurium cells with a TRIzoL reagent (Gibco-BRL) according to the manufacturer's instructions. The primer used for detection of the fliA transcripts was IAP1 (GAGTGTTTATCCATTACAC). Radiolabelling of the primer and the primer extension reaction were carried out according to the procedures described previously (Kutsukake \& Ide, 1995). The extension products were separated on a DNA sequencing gel alongside sequencing ladders of the $\mathrm{fliA}_{\mathrm{A}}$ promoter region made using the same primer.

In vitro transcription. In vitro RNA synthesis was performed using $E$. coli RNA polymerase according to the method described by Kajitani \& Ishihama (1983) with the following modifications. The reaction mixture contained $50 \mathrm{mM}$ Tris/ $\mathrm{HCl}, \mathrm{pH} 7 \cdot 8,50 \mathrm{mM} \mathrm{NaCl}, 0 \cdot 1 \mathrm{mM}$ EDTA, $0 \cdot 1 \mathrm{mM}$ DTT, $25 \mu \mathrm{g} \mathrm{BSA} \mathrm{ml}^{-1}, 3 \mathrm{mM}$ magnesium acetate, $150 \mu \mathrm{M}$ each ATP, GTP and CTP, $50 \mu \mathrm{M}$ UTP with $10 \mu \mathrm{Ci}\left(3.7 \times 10^{5} \mathrm{~Bq}\right)[\alpha-$ ${ }^{32}$ P]UTP (ICN), $1 \mu \mathrm{g}$ template DNA and $1.5 \mu \mathrm{g}$ RNA polymerase. Mixtures without substrates were prepared in a $35 \mu \mathrm{l}$ volume and incubated at $37^{\circ} \mathrm{C}$ for $30 \mathrm{~min}$. The reaction was started by adding $15 \mu$ l prewarmed substrate mixture. After incubation at $37^{\circ} \mathrm{C}$ for $10 \mathrm{~min}$, the reaction was stopped by adding $50 \mu \mathrm{l}$ stop solution $(0.6 \mathrm{M}$ sodium acetate, $\mathrm{pH} 5 \cdot 5$, $20 \mathrm{mM}$ EDTA, $200 \mu \mathrm{g}$ tRNA $\mathrm{ml}^{-1}$ ). The transcripts were extracted with phenol and precipitated with ethanol. The precipitated materials were dissolved in $20 \mu$ loading solution ( $8 \mathrm{M}$ urea, $0.025 \%$ bromophenol blue, $0.025 \%$ xylene cyanol) and electrophoretically separated in a $4 \%$ polyacrylamide gel containing $8 \mathrm{M}$ urea. The transcript bands were visualized by autoradiography on an X-ray film.

DNA sequencing and PCR. DNA sequences were determined by the dideoxy chain-termination method with an automated DNA sequencer model ABI $373 \mathrm{~S}$ (Perkin Elmer) using various synthetic primers. Sequence data were analysed by use of the software GENETYX (Software Development). PCR was performed using Taq DNA polymerase according to the manufacturer's instructions.

Northern blot hybridization. Total RNA was isolated from S. typhimurium cells as described above, and $20 \mu \mathrm{g}$ of each (a)

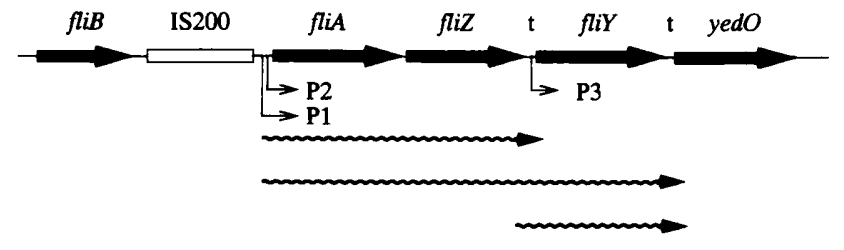

(b)

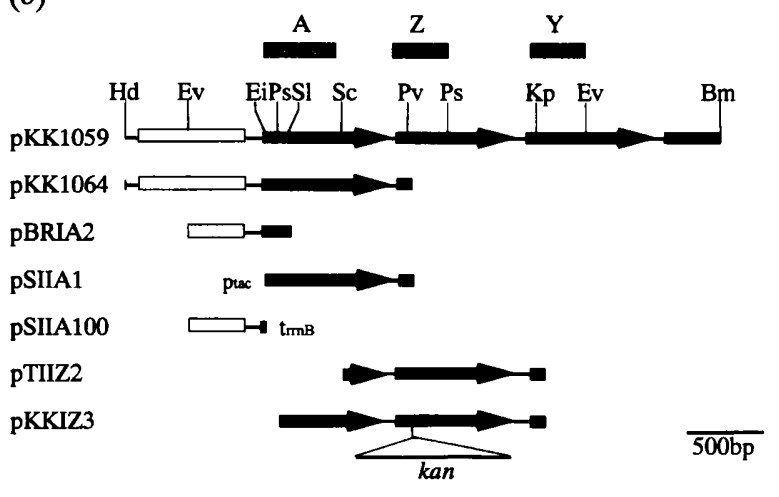

Fig. 1. Structure and transcriptional organization of the fliA operon of S. typhimurium (a) and the chromosomal regions cloned on the recombinant plasmids (b). Only restriction sites used in the present study are shown: $\mathrm{Bm}, \mathrm{BamHI}$; Ei, EcoRI; Ev, EcoRV; Hd, HindIII; Kp, Kpnl; Ps, Pstl; Pv, Pvull; Sc, Sacl; Sl, Sall. The probes used in the Northern blotting analysis are indicated by hatched bars. P1, P2 and P3 indicate the transcription start sites, and wavy arrows represent RNA transcripts. $t$, Possible transcription-termination site; $k a n, k a n$ gene cassette; $p_{t a c}$ and $t_{r r n B}$, tac promoter and the $r r_{B} B$ terminator, respectively.

sample was separated electrophoretically on an agarose gel. DNA probes used were probes $\mathrm{A}, \mathrm{Z}$ and $\mathrm{Y}$, which are internal to the $f l i A, f l i Z$ and $f l i Y$ genes, respectively (Fig. 1). Probes A and $\mathrm{Y}$ were made by excising the $0.6 \mathrm{~kb}$ EcoRI-SacI and $0.4 \mathrm{~kb}$ KpnI-EcoR V fragments from pKK1059, respectively. Probe Z was prepared by excising the $0.4 \mathrm{~kb}$ BamHI-PstI fragment from the amplified product by PCR using primers IZP1 (GGGGATCCACGGTGCAGCAACCTAAAAG) and IYP3 (GGGAATTCATAGTTAGTCCCCAGCCCAA) and pKK1059 as a template. These probes were radiolabelled with a BcaBEST labelling kit (Takara) and $\left[\alpha^{32} \mathrm{P}\right] \mathrm{dCTP}$ (ICN) according to the manufacturer's instructions. RNA blotting and hybridization were performed according to the method of Baga et al. (1985). Radioactive bands were visualized using a BAS2000 image analyser (Fuji).

Construction of a fliz-disruption mutant. The $0.84 \mathrm{~kb}$ Pst IPvuII fragment excised from pKK1064 was inserted into pUC19 at the PstI/HinclI site to yield pUCIZ1. The $0.79 \mathrm{~kb}$ PvuII-KpnI fragment excised from pKK1059 was inserted into pUCIZ1 at the $S m a I / K p n I$ site to yield pKKIZ2. The kanamycin-resistance gene cassette ( $k a n)$ excised from pUC4K with $B a m \mathrm{HI}$ was inserted into pKKIZ2 at the BamHI site to yield pKKIZ3. This plasmid carries the kan gene inserted into the $f l i Z$ gene. The chromosomal fliZ gene of a wild-type strain, KK1004, was replaced with this fliZ::kan allele according to the method described previously (Kutsukake, 1994; Kutsukake et al., 1994a). The structure of the chromosomal fliZ gene was examined by Southern blot analysis with a DIG DNA labelling and detection kit (Boehringer Mannheim). One 
clone with the desired structure was named KK1397 and used in this study.

\section{RESULTS AND DISCUSSION}

\section{Primer extension analysis of the fliA gene}

In order to identify the promoters of the fliA gene, primer-extension-mediated mapping of the transcriptional start sites was carried out. RNAs were isolated from KK1004 (wild-type), KK2040 (flhD::Tn10) and KK2091 (fliA:: Tn10) harbouring pBRIA2, which carries the promoter region of the fliA gene. In the RNA sample from KK1004, two positive signals were observed (Fig. 2 ). They map to adenine nucleotides positioned 29 and 18 bases upstream of the initiation codon of the fliA gene. These start sites were designated P1 and P2, respectively.

The P1 signal was absent in the RNA sample from KK2040 but present in that from KK2091. This indicates that the P1 signal is dependent on FlhD/FlhC but not on FliA. Therefore, we conclude that $\mathrm{P} 1$ is a start site from a class 2 promoter. The sequence around the -10 region of $\mathrm{P} 1$ resembles the -10 consensus sequence of the $\sigma^{70}$ dependent promoter, but a sequence corresponding to the -35 region is not evident. Instead, the sequence TTATTCC, which is conserved in the upstream regions of the class 2 promoters of S. typhimurium (Kutsukake \& Ide, 1995), is present at the region centred $42 \mathrm{bp}$ upstream of P1. The DNA sequence to which the FlhD/FlhC complex binds was reported in the E. coli fliA promoter (Liu \& Matsumura, 1994). It corresponds to the DNA region $30-80$ bp upstream of $\mathrm{P} 1$. Within this region, the sequence AAATAACCC, centred $63 \mathrm{bp}$ upstream of $\mathrm{P} 1$, is exactly conserved between $E$. coli and $S$. typhimurium. This suggests that these nine nucleotides may be responsible for FlhD/FlhC binding.

The P2 signal was absent in both RNA samples from KK2040 and KK2091. This indicates that P2 is dependent on both FlhD/FlhC and FliA. Because the fliA gene is under positive control of FlhD/FlhC (Kutsukake et al., 1990), we conclude that P2 is a start site from a class 3 promoter. Consistent with this, both the -10 and -35 regions of $\mathrm{P} 2$ are homologous to the -10 and -35 consensus sequences of the class 3 promoter (Arnosti \& Chamberlin, 1989; Kutsukake et al., 1990). These results agree with our previous observation that the fliA gene is under positive control of its own product (Kutsukake $\&$ lino, 1994).

\section{In vitro transcription of the fliA gene}

In order to confirm that the promoters identified above are actually class 2 and class 3 promoters, in vitro transcription analysis of the fliA gene was performed using purified transcription factors (Fig. 3). The DNA template used was a supercoiled DNA of pSIIA100, in which the fliA promoter region is located about $350 \mathrm{bp}$ upstream of the terminator sequence of the $r r n B$ operon. When core enzyme or $\sigma^{70}$-holoenzyme was used without other transcription factors, no transcription occurred. (a)

G A $\begin{array}{llllll}\text { A } & \text { T } & \text { C } & 1 & 2 & 3\end{array}$

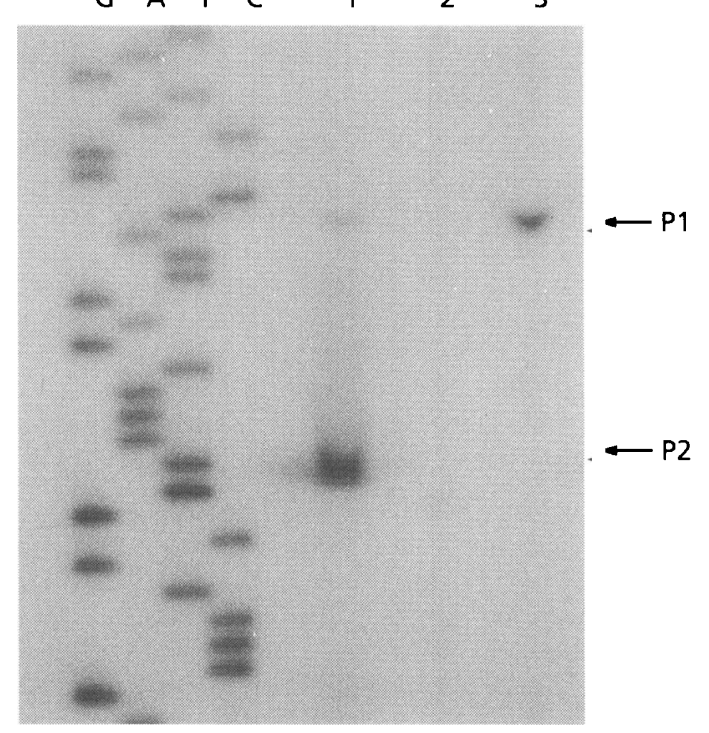

(b)

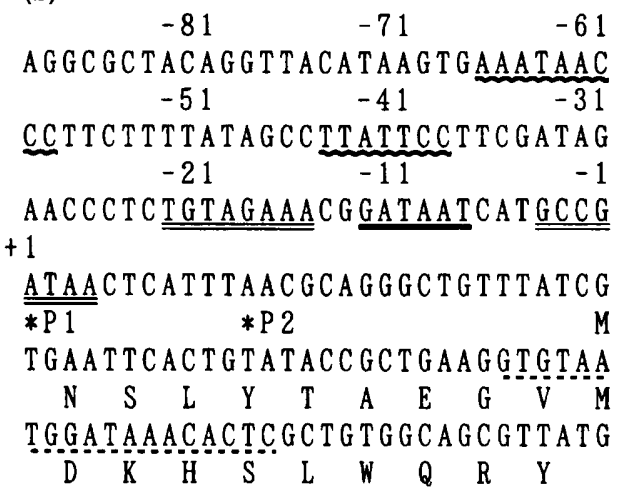

Fig. 2. Transcriptional mapping of the fliA operon by primer extension analysis (a) and the structure of the fliA promoter region (b). (a) RNAs were isolated from KK1004 (lane 1), KK2040 (lane 2) and KK2091 (lane 3). The transcription start sites were identified by primer extension with primer IAP1. The sequencing ladder (lanes $G, A, T$ and $C$ ) was made with the same primer. P1 and P2 indicate the class 2 and class 3 transcription start sites, respectively. (b) The sequence complementary to the primer used is indicated by a broken line. The $P 1$ start site is labelled as +1 . The -10 region of $P 1$ is underlined, while the -10 and -35 regions of $P 2$ are doubly underlined. The sequences conserved in other class 2 promoters of S. typhimurium and in the E. coli fliA promoter are indicated by wavy lines. Sequence data were taken from Ohnishi et al. (1990).

When $\sigma^{70}$-holoenzyme was used with FlhD/FlhC, one transcript of about 350 nucleotides was produced. This is a class 2 transcript and must correspond to the P1 transcript. When core enzyme or $\sigma^{70}$-holoenzyme was used with FliA, one slightly shorter transcript was produced. This is a class 3 transcript and must correspond to the P2 transcript. These were further confirmed by determining the transcription start sites of the in vitro transcripts by primer extension analysis (data not shown). 


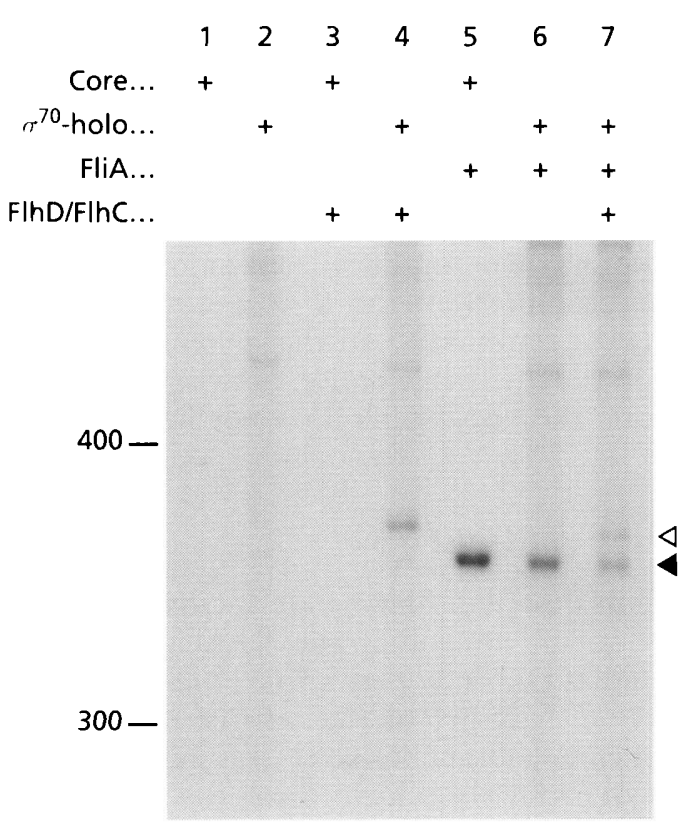

Fig. 3. In vitro transcription of the fliA operon. The DNA template used was a supercoiled DNA of pSIIA100, which carries the class 2 and class 3 fliA promoters located about $350 \mathrm{bp}$ upstream of the $r$ rnB terminator on pTrc97A (Fig. 1b). Transcription factors added in the reaction mixture are indicated above each lane: core, RNA polymerase core enzyme; $\sigma^{70}$-holo, $\sigma^{70}$-containing RNA polymerase holoenzyme; FliA, FliA protein; FlhD/FlhC, FlhD/FlhC complex. All the reactions were carried out using $1.5 \mu \mathrm{g} E$. coli RNA polymerase. When required, $40 \mathrm{pmol}$ of the FliA protein or the FlhD/FlhC complex was added to the reaction mixture, except for lane 7 . In the reaction mixture of lane $7,10 \mathrm{pmol}$ FliA and $40 \mathrm{pmol}$ FlhD/FlhC were added simultaneously. Open and filled triangles indicate the class 2 and class 3 transcripts, respectively. Positions of RNA size markers are indicated in nucleotides on the left.

\section{Sequence analysis of the region downstream of the fliA gene}

Plasmid pKK1059 contains the fliA gene and its adjacent region of the $S$. typhimurium chromosome (Fig. 1). Ohnishi et al. (1990) reported the DNA sequence of the $1.4 \mathrm{~kb}$ EcoRV-Pvull fragment of this plasmid. The reported sequence contains the fliA gene and its downstream region of $0.2 \mathrm{~kb}$. Examination of this downstream sequence revealed that it contains the $5^{\prime}$ portion of an ORF (orf1) which starts from either of the ATG codons 8 bp and $59 \mathrm{bp}$ downstream of the termination codon of the fliA gene and extends to the PvuIl site. Because there is no potential transcription-termination signal in the intergenic region, orf1 is likely to be transcribed together with fliA.

In order to know the entire structure of the $f l i A$ operon, we determined the sequence of the PvuII-BamHI fragment of pKK1059 (accession no. AB010947). It was found that orf1 is followed by two additional ORFs, orf 2 and orf3. orf 2 starts from either of the ATG codons $99 \mathrm{bp}$ and $147 \mathrm{bp}$ downstream of the termination codon of orf1. The intergenic region between orf 1 and orf 2 contains sequences similar to the -35 and -10 consensus sequences of the $\sigma^{70}$-dependent promoter, suggesting that orf 2 may be transcribed by $\sigma^{70}$-RNA polymerase. Two pairs of inverted repeats which may function as the transcriptional terminators are present in the downstream region of orf 2 . orf 3 starts from the ATG codon $154 \mathrm{bp}$ downstream of the termination codon of orf2 and extends to the BamHI site.

As expected, the determined sequence is homologous to the previously sequenced E. coli fliAZY locus (Mytelka \& Chamberlin, 1996; Itoh et al., 1996). The deduced amino acid sequences of Orf1, Orf2 and Orf3 show homology to FliZ, FliY and YedO of E. coli, respectively. In all cases, they are more than $80 \%$ identical in amino acid sequence in these two bacteria. Therefore, we conclude that orf1, orf 2 and orf 3 are the $S$. typhimurium counterparts of the fliZ, fliY and yedO genes, respectively (Fig. 1). In the fliZ and fliY genes, deduced amino acids between the first and second ATG codons are very diverse both in number and in sequence between $E$. coli and S. typhimurium. This suggests that the second ATG codons are more plausible translation start sites in these two genes. Consistent with this, the second ATG codon of the fliZ gene has a much better match for the ribosome-binding site. If this inference is correct, fliZ and $f l i Y$ should encode proteins of 183 and 266 amino acids, respectively, in both bacteria.

\section{Northern blot analysis of the fliA operon}

Transcriptional organization of the fliAZY locus of $S$. typhimurium was examined by Northern blot analysis using probes $\mathrm{A}, \mathrm{Z}$ and $\mathrm{Y}$, which are internal to the fliA, $f l i Z$ and fliY genes, respectively (Fig. 4). RNAs used were isolated from KK1004 (wild-type) and KK2040 $(f l h D:: \operatorname{Tn} 10)$. When probe $\mathrm{A}$ or probe $\mathrm{Z}$ was used, two major transcripts of about 2300 and 1400 nucleotides
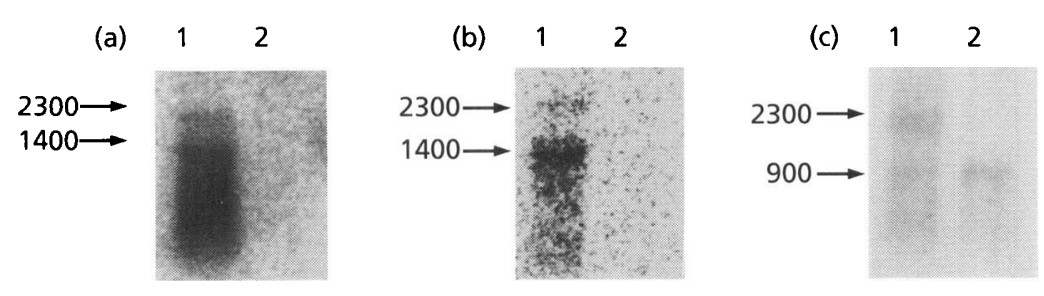

Fig. 4. Northern blot analysis of the fliA, fliz and fliY genes. RNAs were isolated from KK1004 (lane 1) and KK2040 (lane 2). Probes used were probe $A(a)$, probe $Z$ (b) and probe $Y(c)$. Molecular sizes (in nucleotides) are indicated on the left. 


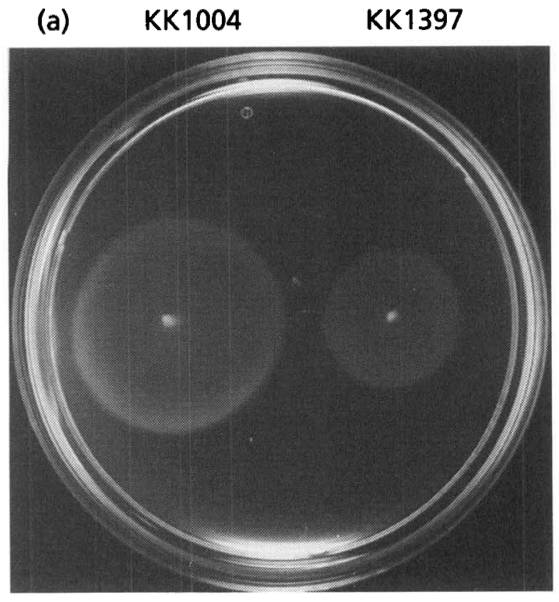

(b)

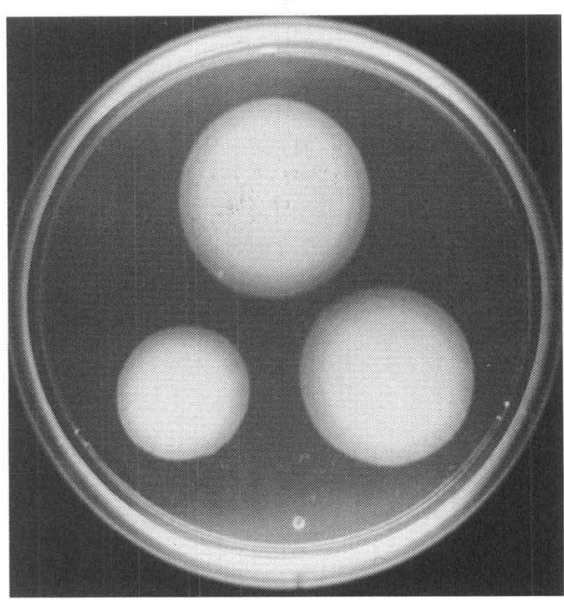

KK1397(pSTV29) KK1397(pTIIZ2)

Fig. 5. Motility phenotype of the fliz::kan mutant. (a) Single colonies of KK1004 (left) and KK1397 (right) were stabbed onto a motility agar plate and incubated for $5 \mathrm{~h}$ at $30^{\circ} \mathrm{C}$. (b) Single colonies of KK1004 harbouring pSTV29 (top) and KK1397 harbouring PSTV29 (bottom, left) or pTIIZ2 (bottom, right) were stabbed onto a motility agar plate containing chloramphenicol and incubated as above.

were detected in RNAs from KK1004. Because neither of these transcripts was detected in RNAs from KK2040, these must be class 2 and/or class 3 transcripts. When probe $\mathrm{Y}$ was used, two major transcripts of about 2300 and 900 nucleotides were detected in RNAs from KK1004. In RNAs from KK2040, the longer transcript was not observed, while the shorter one was still detected. Therefore, the 900-nucleotide transcript is not under control of the flagellar regulon. Together with the data from the transcription start site analysis described above, we conclude that the 2300 - and 1400-nucleotide transcripts correspond to the cotranscription products of the $f l i A, f l i Z$ and $f l i Y$ genes and the $f l i A$ and $f l i Z$ genes, respectively. These are likely to start from the $f l i A$ promoters identified above. The signal of the 2300nucleotide transcript was much weaker than that of the 1400 -nucleotide transcript, indicating that most of the transcripts of the $f i A$ operon terminate between the $f l i Z$ and $f l i Y$ genes. On the other hand, the 900-nucleotide transcript may correspond to a single cistron transcript of the fliY gene, which is likely to start from the $\sigma^{30}$-type promoter residing in the intergenic region between fliZ and $f i \mathrm{Y}$. Therefore, the $f l i \mathrm{Y}$ gene may be transcribed both from its own non-flagellar promoter and from the flagellar promoters of the $f i A$ operon. Transcription presumably terminates at the potential termination signals in the intergenic region between $f l Y$ and yedO. Therefore, the yed $\mathrm{O}$ gene does not belong to the $\mathrm{fliA}$ operon.

\section{Disruption of the fliz gene}

The results described above indicate that at least the fliZ gene is under control of the flagellar regulon. This suggests that FliZ may play a role in flagellar formation or function. Motility of the cells of the polar, Tn10insertion fliA mutant KK2091 was restored by introduction of pKK1064, which carries the fliA gene but not the fliZ gene (data not shown). This indicates that the fliZ gene is not essential for motility development. Next, we constructed a fliZ-disruption mutant of S. typhimurium according to the procedures described in Methods. The obtained mutant, KK1397, formed smaller swarms than the wild-type strain KK1004 on a motility agar plate at $30^{\circ} \mathrm{C}$ (Fig. 5). This effect was reversed by introducing pTIIZ2, which carries the $f$ liZ gene but not the fliY gene (Fig. 5). These results indicate that FliZ is required for expression of maximal motility in S. typhimurium.

In our earlier studies on the flagellar regulon of $S$. typhimurium (Kutsukake et al., 1990; Kutsukake \& Iino, 1994; Kutsukake, 1997b), we used exclusively strain KK2091 (fliA:: Tn10) as a fliA mutant. In this study, we show that the $f l i A$ operon includes the $f l i Z$ gene. This indicates that the $f l Z$ gene is not expressed efficiently in KK2091 owing to a polar effect of the Tn10 insertion. Because FliZ is likely to play a role in flagellar formation or function, it is possible that at least one or some of the observed effects of the fliA: : Tn10 mutation could be attributed to a lowered expression of the $f i z$ gene. Experiments are now in progress to test this possibility.

\section{Function of the fliY and yedO genes}

Mytelka \& Chamberlin (1996) reported that a fliZYdeletion mutant of $E$. coli showed weak motility. We show here that the fliZ mutant which carries an intact fliY gene also exhibited weak motility. This suggests that the impaired motility of the E. coli fliZY-deletion mutant may be caused by a defect of the fliZ gene. Based on the sequence homology of FliY with extracellular solute-binding proteins, Mytelka \& Chamberlin (1996) proposed a hypothesis that E. coli FliY may modulate motility of the cell through binding a certain amino acid or amino acid analogue in the periplasmic space. We show here that the S. typhimurium fliY gene is transcribed from a non-flagellar promoter immediately up- 
stream of the gene. This raises the possibility that the fliY gene may not be a member of the flagellar genes. Of course, we cannot exclude the possibility that this gene belongs to the flagellar genes, because the Northern blot analysis with probe $\mathrm{Y}$ (Fig. 4) suggests that the fliA flagellar and $f l i Y$ non-flagellar promoters may contribute almost equally to $f l i Y$ transcription in the wild-type cells. In order to test these possibilities, we are currently attempting to construct a fliY-disruption mutant.

According to the E. coli genome project (Itoh et al., 1996), yedO is registered as a gene encoding a protein homologous to 1-aminocyclopropane-1-carboxylate (ACC) deaminase of Pseudomonas. This enzyme is involved in degradation of ACC, a precursor of the phytohormone ethylene (Campbell \& Thomson, 1996), which is unlikely to be related to flagellar formation and function. The upstream region of the yed $\mathrm{O}$ gene does not contain sequences homologous to any flagellumrelated promoters. These facts suggest that this gene may not be a member of the flagellar genes. At present, the function and expression of the yed $O$ gene remain unknown.

\section{Concluding remarks}

Through in vivo and in vitro studies on the transcription of the fliA operon of S. typhimurium, we showed that this operon is under dual positive control from FlhD/ FlhC and from FliA. Nucleotide sequence and Northern blot analyses revealed that the fliA operon includes two additional genes, $f l i Z$ and $f l i Y$, though the $f l i Y$ gene is also transcribed from its own non-flagellar promoter. A fliZ-disruption mutant showed poor motility, suggesting that this gene, though not essential, is required for expression of maximal motility.

\section{ACKNOWLEDGEMENTS}

Radioisotope facilities were provided by the Center for Gene Science, Hiroshima University. This work was supported by a Grant-in-Aid for Scientific Research from the Ministry of Education, Science and Culture of Japan.

\section{REFERENCES}

Amann, E., Ochs, B. \& Abel, K.-J. (1988). Tightly regulated tac promoter vectors useful for the expression of unfused and fused proteins in Escherichia coli. Gene 69, 301-315.

Arnosti, D. N. \& Chamberlin, M. J. (1989). Secondary $\sigma$ factor controls transcription of flagellar and chemotaxis genes in Escherichia coli. Proc Natl Acad Sci USA 86, 830-834.

Baga, M., Goransson, M., Normark, S. \& Uhlin, B. E. (1985). Transcriptional activation of a Pap pilus virulence operon from uropathogenic Escherichia coli. EMBO J 4, 3887-3893.

Bolivar, F., Rodriguez, R. L., Greene, P. J., Betlach, M. C., Heyneker, H. L., Boyer, H. W., Crosa, J. H. \& Falkow, S. (1977). Construction and characterization of new cloning vehicles. II. A multipurpose cloning system. Gene 2, 95-113.

Campbell, B. G. \& Thomson, J. A. (1996). 1-Aminocyclopropane1-carboxylate deaminase genes from Pseudomonas strains. FEMS Microbiol Lett 138, 207-210.
Chadsey, M. S., Karlinsey, J. E. \& Hughes, K. T. (1998). The flagellar anti- $\sigma$ factor FlgM actively dissociates Salmonella typhimurium $\sigma^{28}$ RNA polymerase holoenzyme. Genes Dev 12, 3123-3136.

Chang, A. C. Y. \& Cohen, S. N. (1978). Construction and characterization of amplifiable multicopy DNA cloning vehicles derived from the P15A cryptic miniplasmid. J Bacteriol 134, 1141-1156.

Gillen, K. L. \& Hughes, K. T. (1991). Molecular characterization of flgM, a gene encoding a negative regulator of flagellin synthesis in Salmonella typhimurium. J Bacteriol 173, 6453-6459.

Gillen, K. L. \& Hughes, K. T. (1993). Transcription from two promoters and autoregulation contribute to the control of expression of the Salmonella typhimurium flagellar regulatory gene, flgM. J Bacteriol 175, 7006-7015.

Igarashi, K. \& Ishihama, A. (1991). Bipartite functional map of the E. coli RNA polymerase $\alpha$ subunit : involvement of the C-terminal region in transcriptional activation by cAMP-CRP. Cell $\mathbf{6 5}$, 1015-1022.

Itoh, T., Aiba, H., Baba, T. \& 21 other authors (1996). A 460-kb DNA sequence of the Escherichia coli $\mathrm{K}-12$ genome corresponding to the $40 \cdot 1-50 \cdot 0$ min region on the linkage map. DNA Res 3, 379-392.

lyoda, S. \& Kutsukake, K. (1995). Molecular dissection of the flagellum-specific anti-sigma factor, FlgM, of Salmonella typhimurium. Mol Gen Genet 249, 417-424.

Kajitani, M. \& Ishihama, A. (1983). Determination of the promoter strength in the mixed transcription system: promoters of lactose, tryptophan and ribosomal protein L10 operons from Escherichia coli. Nucleic Acids Res 11, 671-686.

Komeda, Y. (1982). Fusion of flagellar operons to lactose genes on a Mu lac bacteriophage. J Bacteriol 150, 16-26.

Komeda, Y. (1986). Transcriptional control of flagellar genes in Escherichia coli K-12. J Bacteriol 168, 1315-1318.

Komeda, Y., Kutsukake, K. \& lino, T. (1980). Definition of additional flagellar genes in Escherichia coli K-12. Genetics 94, 277-290.

Kutsukake, K. (1994). Excretion of the anti-sigma factor through a flagellar substructure couples flagellar gene expression with flagellar assembly in Salmonella typhimurium. Mol Gen Genet 243, 605-612.

Kutsukake, K. (1997a). Hook-length control of the exportswitching machinery involves a double-locked gate in Salmonella typhimurium flagellar morphogenesis. J Bacteriol 179, 1268-1273.

Kutsukake, K. (1997b). Autogenous and global control of the flagellar master operon, $f h D$, in Salmonella typhimurium. Mol Gen Genet 254, 440-448.

Kutsukake, K. \& Ide, N. (1995). Transcriptional analysis of the flgK and fliD operons of Salmonella typhimurium which encode flagellar hook-associated proteins. Mol Gen Genet 247, 275-281.

Kutsukake, K. \& lino, T. (1994). Role of the FliA-FlgM regulatory system on the transcriptional control of the flagellar regulon and flagellar formation in Salmonella typhimurium. J Bacteriol 176, 3598-3605.

Kutsukake, K., Ohya, Y., Yamaguchi, S. \& lino, T. (1988). Operon structure of flagellar genes in Salmonella typhimurium. Mol Gen Genet 214, 11-15.

Kutsukake, K., Ohya, Y. \& lino, T. (1990). Transcriptional analysis of the flagellar regulon of Salmonella typhimurium. J Bacteriol 172, 741-747.

Kutsukake, K., Okada, T., Yokoseki, T. \& lino, T. (1994a). Sequence analysis of the $f g A$ gene and its adjacent region in Salmonella 
typhimurium, and identification of another flagellar gene, flgN. Gene 143, 49-54.

Kutsukake, K., lyoda, S., Ohnishi, K. \& lino, T. (1994b). Genetic and molecular analysis of the interaction between the flagellumspecific sigma and anti-sigma factors in Salmonella typhimurium. $E M B O J 13,4568-4576$.

Liu, X. \& Matsumura, P. (1994). The FlhD/FlhC complex, a transcriptional activator of the Escherichia coli flagellar class II operons. J Bacteriol 176, 7345-7351.

Liu, X. \& Matsumura, P. (1995). An alternative sigma factor controls transcription of flagellar class III operons in Escherichia coli: gene sequence, overproduction, purification and characterization. Gene 164, 81-84.

Liu, X. \& Matsumura, P. (1996). Differential regulation of multiple overlapping promoters in flagellar class II operons in Escherichia coli. Mol Microbiol 21, 613-620.

Macnab, R. M. (1996). Flagella and motility. In Escherichia coli and Salmonella: Cellular and Molecular Biology, 2nd edn, vol. I, pp. 123-145. Edited by F. C. Neidhardt and others. Washington, DC: American Society for Microbiology.
Mytelka, D. S. \& Chamberlin, M. J. (1996). Escherichia coli fliAZY operon. J Bacteriol 178, 24-34.

Ohnishi, K., Kutsukake, K., Suzuki, H. \& lino, T. (1990). Gene fliA encodes an alternative sigma factor specific for flagellar operons in Salmonella typhimurium. Mol Gen Genet 221, 139-147.

Ohnishi, K., Kutsukake, K., Suzuki, H. \& lino, T. (1992). A novel transcriptional regulation mechanism in the flagellar regulon of Salmonella typhimurium: an anti-sigma factor inhibits the activity of the flagellum-specific sigma factor, $\sigma^{\mathrm{F}}$. Mol Microbiol 6, 3149-3157.

Studier, F. W. \& Moffatt, B. A. (1986). Use of bacteriophage T7 RNA polymerase to direct selective high-level expression of cloned genes. J Mol Biol 189, 113-130.

Yanisch-Perron, C., Vieira, J. \& Messing, J. (1985). Improved M13 phage cloning vectors and host strains: nucleotide sequences of the M13mp18 and pUC19 vectors. Gene 33, 103-119.

Received 2 December 1998; revised 15 February 1999; accepted 24 February 1999. 